\title{
ZEROS OF SUCCESSIVE DERIVATES OF A CLASS OF REAL ENTIRE FUNCTIONS OF EXPONENTIAL TYPE
}

\author{
LI-CHIEN SHEN
}

\begin{abstract}
Using the method of steepest descent we prove that for a class of real entire functions of exponential type $\tau$ the spacings of the adjacent zeros of $f^{(n)}$ converge to $\pi \tau / 2$.
\end{abstract}

1. Introduction. Following Pólya, we say that the point $z_{0}$ belongs to the final set of $f$, if

(1) $f$ is analytic at $z_{0}$;

(2) for every $\varepsilon>0$, the disk $\left|z-z_{0}\right|<\varepsilon$ contains zeros of infinitely many functions of the sequence $\left\{f^{(n)}\right\}_{n=1}^{\infty}$.

We say that an entire function $f$ is real if it takes real values for real $z$.

In an address presented to the American Mathematical Society (April 3, 1942), Pólya asserted that $[4$, p. 181]:

"For certain entire functions of order 1 , as, for example, for $f(z)=\sin z$ the differentiation does not change essentially the density of the distribution of the zeros."

In view of the above general assertion of Pólya and the other examples considered by R. Boas and C. Prather [2], the following conjecture seems plausible.

HYPOTHETICAL THEOREM. Let $f$ be a real entire function of order 1, exponential type, and bounded on the real axis. Then the final set of $f$ is an equally spaced infinite set $\{n \pi \tau / 2\}_{-\infty}^{\infty}$, where $\tau$ is the type of $f$.

Recently, C. Prather [5] published a theorem which confirms the Hypothetical Theorem. However, his proof contains an error. In fact, the error is serious, since M. Rao and the author have proved the following theorem which disproves the above Hypothetical Theorem.

THEOREM A [6]. There exists a real entire function of exponential type, of order 1 , and bounded on the real axis such that the entire real axis belongs to its final set.

The function $F$ mentioned in Theorem $\mathrm{A}$ is of the form

$$
F(z)=\int_{0}^{1} \cos z t d \lambda+\frac{1-\cos z}{z},
$$

where $\lambda$ is a finite nonnegative measure.

It must be pointed out here that Theorem A does not contradict Pólya's assertion, for Pólya only stated that "the density" of the distribution of the zeros of

Received by the editors November 25, 1985 and, in revised form, April 1, 1986.

1980 Mathematics Subject Classification (1985 Revision). Primary 30A66; Secondary 30 A64.

Key words and phrases. Real entire function, exponential type, final set. 
$f^{(n)}$ does not change essentially. In our constructed function $F$, the spacings of the zeros of a subsequence of $\left\{F^{(n)}\right\}$ do coverge to $\pi / 2$, but the zeros are "drifting" around the real axis to fill up the whole real axis. Therefore, the assertion of Pólya deserves further investigation. We begin this investigation by considering a real entire function of order 1 , exponential type, and having the following properties.

(i) The type $\tau$ of $f$ is $>0$,

(ii) there is a sector $S$ containing the imaginary axis, that is,

$$
S=\{z:|\arg z \pm \pi / 2| \leq c\} \quad(0<c<\pi / 2)
$$

such that $f$ has only finitely many zeros in $S$;

(iii)

$$
\int_{-\infty}^{\infty} \frac{|\ln | f(t)||}{1+t^{2}} d t<\infty
$$

The above function is, in many aspect, the closest "relative" of $\sin z$, and the purpose of this work is to study the successive derivatives of this function. We will prove the following weaker version of the Hypothetical Theorem.

THEOREM 1. Let $f$ be a real entire function of order 1 and satisfy properties (i), (ii), and (iii). If $f$ is even, then the final set of $f$ is $\{n \tau \pi / 2\}_{n=-\infty}^{\infty}$.

In $\S 2$, the outline of our method is presented. All the needed lemmas are stated in $\S \S 3$ and 4 and in $\S 5$ we prove Theorem 1 . The main finding is presented in $\S 6$.

2. Outline of the method. throughout the paper $z$ is a complex variable which belongs to a fixed but otherwise arbitrary compact set in the complex plane. $D(w, r)$ will denote the disk centered at $w$ with radius $r$.

For an analytic function $f$, we define

$$
\begin{gathered}
a(z)=z f^{\prime} / f \\
b(z)=z(a(z))^{\prime}=z f^{\prime} / f+z^{2}\left(f^{\prime} / f\right)^{\prime} .
\end{gathered}
$$

We will use the method of steepest descent on the integral

$$
f^{(n)}=\frac{n !}{2 \pi i} \int_{|s|=R} f(s)(s-z)^{-n-1} d s .
$$

to obtain an asymptotic expression for $f^{(n)}$ as $n \rightarrow \infty$. This method requires

(a) the adquate selection of a positive sequence $r_{n}$ with $r_{n} \rightarrow \infty$;

(b) the choice of a point $\zeta_{n}=r_{n} e^{i \theta_{n}}$ such that $a\left(\zeta_{n}\right)=n$ for each $n$;

(c) the use of the following lemma which enables us to obtain an accurate approximate of $f$ around the point $\zeta_{n}$.

LEMMA A [3, p. 78]. Let $f$ be a function which is analytic and has no zeros in the disk $D(w, \rho|w|), 0<\rho<1$. Let $a(z)$ and $b(z)$ be defined as above. If there exists a constant $k$ such that

$$
|b(z)| \leq k|b(w)| \quad \text { for all } z \in D(w, \rho|w|),
$$

then

and

$$
\log f\left(w e^{i \theta}\right)=\log f(w)+i \theta a(w)-\theta^{2} b(w) / 2+E(w, \theta)
$$

$$
|E(w, \theta)| \leq k\left|b(w) \theta^{3}\right| / \rho \quad \text { for }|\theta| \leq \rho / 2
$$


In view of Lemma $\mathrm{A}$, it is essential that we understand the properties of $a(z)$ and $b(z)$; we therefore begin our investigation by studying the logarithmic derivative of $f$.

3. The asymptotic behavior of $f^{\prime} / f$ as $z \rightarrow \infty$. Let

$$
S_{0}=\left\{z:|\arg z \pm \pi / 2| \leq c_{1}<c\right\} \text {. }
$$

Clearly, $S_{0} \varsubsetneqq S$. We use $S_{0}^{+}$and $S_{0}^{-}$to denote the portions of $S_{0}$ lying on the upper half-plane and the lower half-plane, respectively.

We first establish

LEMMA 3.1. Let $f$ be a real entire function of exponential type satisfying properties (i), (ii), and (iii). Then

$$
f^{\prime} / f \rightarrow-i \tau \quad \text { uniformly as } z \rightarrow \infty \text { in } S_{0}^{+},
$$

and

$$
f^{\prime} / f \rightarrow i \tau \quad \text { uniformly as } z \rightarrow \infty \text { in } S_{0}^{-} \text {. }
$$

Proof. Since $f$ is of type $\tau$ and satisfies (iii), it follows that [1, p. 92], for $z$ in the upper half-plane,

$$
\log |f(z)|=\log |B(z)|+\frac{y}{\pi} \int_{-\infty}^{\infty} \frac{\log |f(t)|}{(t-x)^{2}+y^{2}} d t+\tau y
$$

$(z=x+i y)$, where $B(z)$ is the Blaschke product formed from the zeros of $f$, that is,

$$
B(z)=\prod_{n}\left(1-z / z_{n}\right) /\left(1-z / \bar{z}_{n}\right),
$$

where $z_{n}$ are zeros of $f$ on the upper half-plane.

Let $G(z)=f(z) / B(z)$. Then the integral in (3.1) is the real part of the function $\log G(z)-i \tau z$ which is well defined and analytic in the upper half-plane. We now note that

$$
G^{\prime} / G=f^{\prime} / f-B^{\prime} / B
$$

and

$$
\begin{aligned}
\operatorname{Re} \frac{G^{\prime}}{G} & =\frac{\partial}{\partial x} \log |G(z)| \\
& =\frac{2 y}{\pi} \int_{-\infty}^{\infty} \frac{(t-x) \log |f(t)|}{\left((t-x)^{2}+y^{2}\right)^{2}} d t \\
& =\operatorname{Re}\left\{\frac{-i}{\pi} \int_{-\infty}^{\infty} \frac{\log |f(t)|}{(t-z)^{2}} d t\right\}
\end{aligned}
$$

Therefore,

$$
\frac{G^{\prime}}{G}=-\frac{i}{\pi} \int_{-\infty}^{\infty} \frac{\log |f(t)|}{(t-z)^{2}} d t+i k
$$

where $k$ is a real constant and we will see that $k$ is equal to $-\tau$.

From (iii), we see that

$$
G^{\prime} / G \rightarrow i k
$$


as $z \rightarrow \infty$ on the upper and lower half-planes. In fact, for any $c>0$, the convergence is uniform inside the region $c \leq|\arg z| \leq \pi-c$.

And since (iii), implies [1, p. 86]

$$
\sum\left|\operatorname{Im} \frac{1}{z_{n}}\right|=\sum \frac{y_{n}}{\left|z_{n}\right|}<\infty \quad\left(z_{n}=x_{n}+y_{n}\right)
$$

it follows from (3.2) that

$$
B^{\prime} / B=2 i \sum y_{n} /\left(z-z_{n}\right)\left(z-\bar{z}_{n}\right) \rightarrow 0
$$

uniformly as $z \rightarrow \infty$ in $S_{0}^{+}$.

From (3.3), (3.5), and (3.6), we have

$$
f^{\prime} / f \rightarrow i k \quad \text { uniformly as } z \rightarrow \infty \text { in } S_{0}^{+} \text {. }
$$

But this will imply that

$$
\frac{\log \left|f\left(r e^{i \theta}\right)\right|}{r} \rightarrow-k \sin \theta \quad \text { as } z=r e^{i \theta} \rightarrow \infty \text { in } S_{0}^{+} .
$$

And from (3.1), we have

$$
\frac{\log \left|f\left(r e^{i \theta}\right)\right|}{r} \rightarrow \tau \sin \theta
$$

this and (3.8) give $k=-\tau$.

The proof for $z \rightarrow \infty$ in $S_{0}^{-}$is identical, we omit it.

4. Consequences of Lemma $A$ and Lemma 3.1. We let

$$
\begin{aligned}
& S_{1}=\{z:|\arg z \pm \pi / 2| \leq c / 2\}, \\
& S_{2}=\{z:|\arg z \pm \pi / 2| \leq c / 4\} .
\end{aligned}
$$

We now use Lemma 3.1 to derive

LEMMA 4.1. Let $f$ satisfy the assumptions of Lemma 3.1. Then $b(z) / z \rightarrow-i \tau$ uniformly as $z \rightarrow \infty$ in $S_{2}^{+}$.

ProOF. By assumption $f$ has only finitely many zeros in $S$, we can find a positive constant $\rho<1$ so that the disk $D(z, \rho|z|)$ is contained in $S_{1}$ and $f$ has no zeros in it whenever $z \in S_{2}$ and $|z| \geq r_{0}$. Using Cauchy's formula for the derivative, we have

$$
\left(\frac{f^{\prime}}{f}\right)^{\prime}(z)=\frac{1}{2 \pi i} \int_{|s-z|=\rho|z|}\left(\frac{f^{\prime}}{f}(s)+i \tau\right)(s-z)^{-2} d s .
$$

From (4.1) and Lemma 3.1, it is easy to see that

$$
\left|\left(\frac{f^{\prime}}{f}\right)^{\prime}(z)\right|=o\left(|z|^{-1}\right) \quad \text { as } z \rightarrow \infty \text { in } S_{2}^{+} \text {. }
$$

This and Lemma 3.1 immediately yield

$$
b(z)=\frac{z f^{\prime}}{f}+z^{2}\left(\frac{f^{\prime}}{f}\right)^{\prime}=-i \tau z(1+o(1)) \quad\left(z \in S_{2}^{+}\right) .
$$

This complets the proof.

REMARK. It is obvious that $b(z) / z \rightarrow i \tau$ uniformly as $z \rightarrow \infty$ in $S_{2}^{-}$.

The following lemma contains all the essential information to prove Theorem 1 . 
LEMMA 4.2. Let $f$ satisfy the assumptions of Theorem 1. Then

(a) $f\left(i r e^{i \theta}\right)=(1+o(1)) f(i r) \exp \left\{i \theta a(i r)-\theta^{2} b(i r) / 2\right\}$ uniformly as $r \rightarrow \infty$ for $|\theta| \leq \delta(r)$

(b) $f\left(i r e^{i \theta}\right)=o(f(i r)) /\left(b(i r)^{1 / 2}\right)$ uniformly as $r \rightarrow \infty$ for $\delta(r) \leq|\theta| \leq \pi / 2$; where $\delta(r)=b(i r)^{-2 / 5}$.

Proof. From Lemma 4.1, we have

$$
\log f\left(i r e^{i \theta}\right)=\log f(i r)+i \theta a(i r)-\frac{\theta^{2}}{2} b(i r)+E(i r, \theta)
$$

and

$$
|E(i r, \theta)| \leq k\left|b(i r) \theta^{3}\right| / \rho \quad \text { for }|\theta| \leq \rho / 2 .
$$

Since $f$ is even, $a(i r)$ and $b(i r)$ are both real and, from Lemma 4.1, $b(i r)=$ $r(1+o(1))$. Hence, for $|\theta| \leq \delta(r),(4.3)$ implies

$$
|E(i r, \theta)|=O\left(r^{-1 / 5}\right) .
$$

This proves (a).

From (4.2),

$$
\left|f\left(i r e^{i \theta}\right) / f(i r)\right|<\left(1+E^{*}(r, \theta)\right) \exp \left(-\theta^{2} b(i r) / 2\right)
$$

for $|\theta| \leq \rho / 2$, where $\left|E^{*}(r, \theta)\right|<\tilde{k}|\theta|$ and the finite constant $\tilde{k}$ is independent of $r$.

We now select $\theta_{0}$ such that $0<\theta_{0} \leq \rho / 2$ and $1+E^{*}(r, \theta)>1 / 2$. Then, for $\delta(r) \leq|\theta| \leq \theta_{0},(4.4)$ implies

$$
\left|f\left(i r e^{i \theta}\right) / f(i r)\right|<\exp \left(-r^{-1 / 5} / 5\right) \quad \text { as } r \rightarrow \infty .
$$

It is well know that $[1$, p. 115], from (3.1),

$$
\limsup _{r \rightarrow \infty} \log \left|f\left(r e^{i \theta}\right)\right| / r=\tau \sin \theta \quad \text { for } 0 \leq \theta \leq \pi .
$$

Moreover, since $f$ has only finitely many zeros in the sector $S^{+}$,

$$
\lim _{r \rightarrow \infty} \log \left|f\left(r e^{i \theta}\right)\right| / r=\tau \sin \theta \quad \text { if }|\theta-\pi / 2| \leq c .
$$

In particular,

$$
\lim \log |f(i r)| / r=\tau .
$$

(It should be commented here that (4.6), $(4.6)^{\prime}$, and (4.7) are valid for noneven functions as well.)

We now select an $h$ such that $h>0$ and $\tau\left(\sin \theta_{0}-1\right)+h<0$. Clearly, (4.6) and (4.7) imply that

$$
\left|f\left(i r e^{i \theta}\right) / f(i r)\right|<\exp \left\{\left(\tau\left(\sin \theta_{0}-1\right)+h\right) r\right\}
$$

for $\theta_{0} \leq|\theta| \leq \pi / 2$ and all $r$ sufficiently large.

The conclusion (b) now follows from (4.5) and (4.8). 
5. Proof of Theorem 1. Without loss of generality, we assume that the type of $f$ is 1 (the general case is reduced to this special case by consideration of $f(z / \tau)$ ). Before proving the theorem, we make the following preparation.

We first select a sequence $\left\{r_{n}\right\}$ so that

$$
a\left(i r_{n}\right)=n \text {. }
$$

The existence of such a sequence follows from the continuity of $a(i r)$ as a function of $r$ and

$$
a(i r)=i r f^{\prime}(i r) / f(i r)=r(1+o(1)) \rightarrow+\infty \quad \text { as } r \rightarrow \infty .
$$

From (5.1) and (5.2), it follows that

$$
\lim r_{n} / n=1,
$$

and

$$
\left(1-z / r_{n} e^{i \theta}\right)^{-n-1} \rightarrow \exp \left(z e^{-i \theta}\right) \quad \text { as } n \rightarrow \infty,
$$

uniformly for all $z$ in a compact set $D$.

Also from (5.4), there exists a constant $M$ such that

$$
\left(1-z / r_{n} e^{i \theta}\right)^{-n-1}<M
$$

for all $n$ sufficiently large and $z$ in $D$.

From Cauchy's formula for the derivatives, we have

$$
f^{(n)}=\frac{n !}{2 \pi r_{n}^{n}}\left\{\int_{0}^{\pi}+\int_{\pi}^{2 \pi} f\left(r_{n} e^{i \theta}\right) e^{-i n \theta}\left(1-\frac{z}{r_{n} e^{i \theta}}\right)^{-n-1} d \theta\right\}=I(n)+J(n) .
$$

We now write

$$
I(n)=\frac{n !}{2 \pi r_{n}^{n}}\left\{\int_{0}^{\pi / 2-\delta_{n}}+\int_{\pi / 2+\delta_{n}}^{\pi}+\int_{\pi / 2-\delta_{n}}^{\pi / 2+\delta_{n}}\right\}=I(n, 1)+I(n, 2)+I(n, 3),
$$

where $\delta_{n}=\delta\left(r_{n}\right)=b\left(i r_{n}\right)^{-2 / 5}$.

Evidently, from (5.5) and part (b) of Lemma 4.2,

$$
I(n, 1)+I(n, 2)=o\left(n ! f\left(i r_{n}\right) /\left(r_{n}^{n} \sqrt{b\left(i r_{n}\right)}\right)\right) \quad \text { as } n \rightarrow \infty .
$$

We now consider $I(n, 3)$. After making an obvious change of variables and using part (a) of Lemma 4.1, we obtain

$$
\frac{2 \pi r_{n}^{n} I(n, 3)}{n !}=\frac{(-i)^{n} f\left(i r_{n}\right)}{\sqrt{b\left(i r_{n}\right)}} \int_{-t_{n}}^{t_{n}}(1+o(1)) e^{-t^{2} / 2} H_{n}(z, t) d t
$$

where

$$
H_{n}(z, t)=\left(1+\frac{z i}{r_{n}} e^{-i t / \sqrt{b\left(i r_{n}\right)}}\right)^{-n-1}
$$

and $t_{n}=\delta_{n}^{-1 / 4}$.

We observe that, for $|t| \leq t_{n}$,

$$
H_{n}(z, t) \rightarrow e^{-i z} \quad \text { and } \quad t_{n}=n^{1 / 10}(1+o(1)) \quad \text { as } n \rightarrow \infty .
$$


Therefore, the integral in (5.7) converges to

$$
e^{-i z} \int_{-\infty}^{\infty} e^{-t^{2} / 2} d t
$$

which is equal to $\sqrt{2 \pi} e^{-i z}$, as $n \rightarrow \infty$.

Hence, together with (5.6),

$$
I(n) \sim \frac{(-i)^{n} n ! f\left(i r_{n}\right)}{r_{n}^{n} \sqrt{2 \pi b\left(i r_{n}\right)}} e^{-i z}
$$

uniformly for all $z$ in $D$.

Similarly, using the facts that $b(i r)=b(-i r)$ and $a(i r)=a(-i r)$,

$$
J(n) \sim \frac{(i)^{n} n ! f\left(i r_{n}\right)}{r_{n}^{n} \sqrt{2 \pi b\left(i r_{n}\right)}} e^{i z} .
$$

Hence,

$$
f^{(n)}(z)=I(n)+J(n) \sim \frac{2 n ! f\left(i r_{n}\right)}{r_{n}^{n} \sqrt{2 \pi b\left(i r_{n}\right)}} \cos \left(z+\frac{n \pi}{2}\right) .
$$

This clearly shows that the final set of $f$ is $\{n \pi / 2\}_{n=-\infty}^{\infty}$.

6. Conclusions. We now consider a real entire function of order 1 and satisfying properties (i), (ii), and (iii). this function may not be even. Without altering the method of the proof of Theorem 1, we can also obtain an asymptotic formula for $f^{(n)}$. Our finding is summarized as follows.

Let $f$ be real entire, of order 1 , and satisfy properties (i), (ii) and (iii); with no loss of generality, let $\tau=1$. Then,

$$
f^{(n)}(z) \sim \frac{n !}{\sqrt{2 \pi}}\left\{\frac{f\left(\zeta_{n}\right) e^{-i z}}{\zeta_{n}^{n} \sqrt{b\left(\zeta_{n}\right)}}+\frac{f\left(\bar{\zeta}_{n}\right) e^{i z}}{\zeta_{n}^{-n} \sqrt{b\left(\bar{\zeta}_{n}\right)}}\right\}
$$

where $\zeta_{n}$ is a solution of the equation $a(z)=z f^{\prime} / f=n$.

We remark that unlike the even case, the existence of such a $\zeta_{n}$ for each sufficiently large $n$ requires a brief discussion.

Since $z f^{\prime} / f=-i z(1+o(1))$ uniformly as $z \rightarrow \infty$ in the sector $S_{2}^{+}$, we have

$$
\left|z f^{\prime} / f-n+i z+n\right|<|i z+n|
$$

for $z$ belonging to the circle $|z-n i|=n \rho$, where $\rho$ is the same constant which we selected earlier in the proof of Lemma 4.1. By Rouché's theorem, the equation $z f^{\prime} / f=n$ has a unique solution in the disk $D(n i, n \rho)$ and

$$
\zeta_{n}=n i(1+o(1))=r_{n} e^{i \theta_{n}}
$$

where $r_{n} / n \rightarrow 1$ and $\theta_{n} \rightarrow \pi / 2$ as $n \rightarrow \infty$.

After selecting this sequence $\left\{\zeta_{n}\right\}$, we can obtain the following facts by repeating the same argument used in the proof of Lemma 4.2:

$(\text { a })^{\prime} f\left(\varsigma_{n} e^{i \theta}\right) \sim f\left(\varsigma_{n}\right) \exp \left\{i n \theta-\theta^{2} b\left(\varsigma_{n}\right) / 2\right\}$ for $|\theta| \leq\left|b\left(\varsigma_{n}\right)\right|^{-2 / 5}$,

(b) $f\left(\varsigma_{n} e^{i \theta}\right)=o\left(f\left(\varsigma_{n}\right) / b\left(\varsigma_{n}\right)^{1 / 2}\right)$ for $0 \leq \theta+\theta_{n} \leq \pi,|\theta|>\left|b\left(\zeta_{n}\right)\right|^{-2 / 5}$.

Then (6.1) follows from (a)' and (b)' immediately.

If we write

$$
\frac{n ! f\left(\zeta_{n}\right)}{\zeta_{n}^{n} \sqrt{2 \pi b\left(\zeta_{n}\right)}}=R_{n} e^{i \theta_{n}}
$$


Then (6.1) becomes

$$
f^{(n)}(z) \sim 2 R_{n} \cos \left(z+\theta_{n}\right) .
$$

This clearly shows that the spacings of the adjacent zeros of $f^{(n)}$ converge to $\pi / 2$.

\section{REFERENCES}

1. R. Boas, Entire functions, Academic Press, New York, 1954.

2. R. Boas and C. Prather, Final sets for operators on finite Fourier transforms, Houston Math. J. 5 (1979), 29-36.

3. W. K. Hayman, A generalisation of Stirling's formula, J. Reine Angew. Math. 196 (1956), 67-95.

4. G. P6lya, On the zeros of the derivatives of a function and its analytic character, Bull. Amer. Math. Soc. 49 (1943), 178-191.

5. C. Prather, Final set for operators on real entire functions of order one, normal type, Proc. Amer. Math. Soc. 90 (1984), 363-369.

6. M. Rao and Li-Chien Shen, On the final set of a real entire function of exponential type, preprint.

Department of Mathematics, UNiversity of Florida, Gainesville, Florida 32611 